\title{
Evaluation of the diagnostic performance of panfungal polymerase chain reaction assay in invasive fungal diseases
}

\author{
GUO-JUN CAO $^{1}$, ZHI-FANG XING ${ }^{2}$, LI HUA ${ }^{3}$, YU-HUA JI ${ }^{3}$, JIA-BIN SUN $^{3}$ and ZHEN ZHAO ${ }^{4}$ \\ ${ }^{1}$ Department of Laboratory Medicine, Huashan Hospital, Fudan University, Shanghai 200040; ${ }^{2}$ Department \\ of Blood Transfusion, Minhang Hospital, Fudan University, Shanghai 201199; ${ }^{3}$ Department of Clinical \\ Laboratory, Ruijin Hospital, Shanghai Jiao Tong University School of Medicine, Shanghai 200025; \\ ${ }^{4}$ Department of Clinical Laboratory, Minhang Hospital, Fudan University, Shanghai 201199, P.R. China
}

Received October 11, 2016; Accepted June 2, 2017

DOI: $10.3892 /$ etm.2017.5081

\begin{abstract}
Timely diagnosis of invasive fungal diseases (IFDs) is important, as delays in treatment initiation are associated with increased mortality rates. However, early diagnosis of IFDs in immunocompromised patients remains difficult. The conventional diagnostic methods currently used for IFDs are not sufficiently effective. Molecular tests, such as polymerase chain reaction (PCR)-based assays, have great potential to improve the early diagnosis of IFDs due to their sensitivity and specificity. In the present study, the diagnostic performance of panfungal PCR assays in IFD patients who received bone marrow transplantation was evaluated. The results suggested that panfungal PCR assay offered a quick and convenient guide for clinical decision-making by identifying higher numbers of fungal species in comparison with the conventional blood culture method. Furthermore, panfungal PCR assay exhibited a sensitivity of $93 \%$ and a specificity of $71 \%$ in the diagnosis of IFD patients based on the EORTC/MSG criteria. Thus, the present study concluded that the reported PCR-based method was effective and sensitive in early IFD diagnosis and should be integrated into clinical decision-making for the treatment of IFDs in the future.
\end{abstract}

\section{Introduction}

The incidence of invasive fungal diseases (IFDs) has increased considerably during the past two decades due to the increasing population of immunocompromised patients, including mainly hematologic malignancy patients and bone marrow transplantation (BMT) or hematopoietic stem cell transplantation recipients (1). Due to the lack of effective

Correspondence to: Dr Zhi-Fang Xing, Department of Blood Transfusion, Minhang Hospital, Fudan University, 170 Xinsong Road, Shanghai 201199, P.R. China

E-mail: xzfisme@163.com

Key words: invasive fungal diseases, panfungal polymerase chain reaction, early diagnosis, blood sample early diagnostic methods, which consequently delays the initiation of therapy, the overall survival rate for immunocompromised patients with IFDs remains poor (2). Early diagnosis in these patients is challenging as a result of the complicated clinical symptoms of IFDs, which are difficult to distinguish from symptoms of bacterial or viral infections (3). According to the recommendations issued by the European Organization for Research and Treatment of Cancer/Mycoses Study Group (EORTC/MSG), the current diagnosis of IFDs requires a combination of epidemiological, histopathological, radioclinical and microbiological diagnostic methods (4-6). However, as summarized in previous studies, all these conventional methods for diagnosing IFDs are time consuming and have limited sensitivity and specificity, particularly when performed on blood samples (7-9). Therefore, there is an urgent need in clinical practice to develop faster and more effective diagnostic methods, in order to improve the survival rates of patients with IFDs.

An increasing number of noninvasive diagnostic tools, including molecular techniques, have been proven to improve fungal diagnostics in a faster, more sensitive and specific manner (7). The most commonly used molecular methods include polymerase chain reaction (PCR)-based assays, which have a great potential to facilitate earlier detection of fungal DNA in a broader range of samples, and consequently lead to a faster initiation of antifungal treatment $(8,10)$. Furthermore, the recognition of the fungal pathogens by PCR and sequencing assays is highly sensitive and precise (8). Recently, a preliminary study in a European population reported that panfungal quantitative PCR assay had a low sensitivity (40\%), but high specificity (96\%), positive predictive value (PPV; 88\%) and negative predictive value (NPV; 69\%) in the diagnosis of IFD involving the Aspergillus species (11). The conclusions of this previous study remain to be confirmed by multi-center experimental studies. Due to economic and environmental reasons, the PCR-based method remains a relatively new assay in China and has not been widely used in fungal DNA detection or in the diagnosis of IFDs (12).

Our previous study reported the application of a PCR-based method in the detection of fungal DNA on sputum specimens (13). In the present study, the technical features of panfungal PCR assay performance for the evaluation of blood 
specimens collected from immunocompromised patients were described. Based on the EORTC/MSG criteria, the current study demonstrated a sensitivity of $93 \%$, a specificity of $71 \%$ and a PPV of $91 \%$ in the diagnosis of IFDs, with the Candida species being the major fungal pathogen.

\section{Materials and methods}

Patients and clinical materials. In the current study, immunocompromised hematologic patients reporting and/or depicting respiratory signs or symptoms were considered at risk for IFDs. A total of 46 patients were consecutively enrolled from the Department of Clinical Laboratory of the Shanghai Minhang District Central Hospital (Shanghai, China) during the period between March 2009 and July 2011. The patients included 32 males and 14 females with ages ranging between 15 and 58 years (average age, 34.1 years). The majority of the patients suffered from hematologic malignancy diseases, including 13 cases of acute lymphoblastic leukemia, 18 cases of acute myelogenous leukemia, 5 cases of chronic myelogenous leukemia, 2 cases of chronic myelomonocytic leukemia, 1 case of mixed leukemia, 4 cases of myelodysplastic syndrome, 1 case of Hodgkin's disease, 1 case of acute promyelocytic leukemia and 1 case of lung cancer.

A total of 118 blood specimens (1 ml per sample; 2-3 samples per patient) were prospectively collected. The clinical information and radiologic reports of patients were collected and reviewed, and laboratory examinations were performed according to the IFD diagnostic criteria of the EORTC/MSG (14). Based on the diagnostic certainty, each patient was classified as having proven, probable, possible or no IFD.

Approval for the study was obtained from the Study Research Ethics Committee of Shanghai Minhang District Central Hospital. Full informed consent was obtained from patients or legal guardians of the patients.

DNA extraction. Genomic DNA was extracted from the blood samples using a method described previously (12). Briefly, cell lysates from the blood samples were generated following treatment with Tris-lysis buffer, lysozyme (R\&D Systems, Inc., Minneapolis, MN, USA) and proteinase K (Solarbio Life Sciences, Beijing, China). Subsequent to overnight incubation at $56^{\circ} \mathrm{C}$, the genomic DNA was isolated using the phenol-chloroform method and then purified through a silica gel column, lywallzyme solution $(20 \mathrm{mg} / \mathrm{ml}$ in $0.6 \mathrm{M}$ mannitol; Guangdong Institute of Microbiology, Guangzhou, China) and cetyltrimethylammonium bromide (CTAB; Nanjing Aocheng Chemical Co., Ltd., Nanjing, China). The resultant DNA pellet was washed with $70 \%$ ethanol and dissolved in deionized $\mathrm{H}_{2} \mathrm{O}$.

PCR and sequencing analysis. A PCR assay was developed for the detection and identification of a broad-range of fungi. The PCR reactions were set up in a total volume of $50 \mu 1$, containing $5 \mu \mathrm{l}$ buffer solution (10X), $4 \mu \mathrm{ldNTPs}(2.5 \mathrm{mmol} / \mathrm{l})$, $4 \mu \mathrm{Mg}^{2+}(25 \mathrm{mmol} / \mathrm{l}), 1 \mu \mathrm{l}$ each of the upstream and downstream primers $(10 \mu \mathrm{mol} / 1), 2 \mu \mathrm{l}$ DNA template, $0.5 \mu \mathrm{l} \mathrm{Taq}$ DNA polymerase (5 U/ $\mu$ l; Takara Bio, Inc., Otsu, Japan; DRR001AM) and autoclaved double distilled water $\left(\mathrm{ddH}_{2} \mathrm{O}\right)$. The primers targeted a consensus sequence for the internal transcribed spacer (ITS) of a variety of fungal pathogens, as reported in other studies $(15,16)$. The primers used were as follows: ITS3 forward, 5'-GCATCGATGAAGAACGCA GC-3'; ITS4 reverse, 5'-TCCTCCGCTTATTGATATGC-3'. GAPDH forward, 5'-CCAGGTGGTCTCCTCTGACTT-3'; GAPDH reverse, 5'-GTTGCTGTAGCCAAATTCGTTGT-3'. Amplification was performed on a TaqMan 7900 instrument (Applied Biosystems; Thermo Fisher Scientific, Inc., Waltham, MA,USA) using the following standard protocol: $3 \mathrm{~min}$ at $55^{\circ} \mathrm{C}$, $10 \mathrm{~min}$ at $95^{\circ} \mathrm{C}$, followed by 35 cycles of $15 \mathrm{sec}$ at $95^{\circ} \mathrm{C}, 60 \mathrm{sec}$ at $55^{\circ} \mathrm{C}$ and $40 \mathrm{sec}$ at $72^{\circ} \mathrm{C}$. Next, the mixture was subjected to a further extension at $72^{\circ} \mathrm{C}$ for $7 \mathrm{~min}$. A Candida albicans fungal DNA sample (ATCC 90028; American Type Culture Collection, Manassas, VA, USA) was included as a positive control, while a pair of GAPDH primers was used as a control to exclude inhibitor contamination and the risk of false-negative results from the panfungal PCR assay.

Following amplification, the PCR products were analyzed by electrophoresis (1.5\% agarose gel). The target band, if visible, was collected and purified from the agarose gel and sent to Invitrogen (Thermo Fisher Scientific, Inc., Shanghai, China) for sequence analysis. The results were compared with the Basic Local Alignment Search Tool database (http://blast.ncbi.nlm.nih.gov/Blast.cgi).

Microbiological culture. The Fungi-Fluor staining solution (Polysciences, Inc., Warrington, PA, USA) was used to analyze clinical specimens by fungal culture (13). Briefly, blood samples were placed in a sterile $0.9 \%$ sodium chloride solution following retrieval and incubated on CHROMagar plates at $35^{\circ} \mathrm{C}$ (CHROMagar Co., Paris, France). The agar plates were examined for growth in the following 7 days. Cultures were considered negative if no fungal growth was visible after 14 days of incubation.

Statistical analysis. Following electrophoresis, the gels of PCR products of all specimens were photographed, and the images were processed using a Gel Logic 200 imager and Kodak molecular imaging software (Kodak Scientific Imaging Systems, Rochester, NY, USA). The intensity of each target band was calculated and expressed as the optical density (OD), and then the receiver operating characteristic (ROC) curve was plotted. The cut-off OD value for the diagnosis of IFD, as well as the corresponding sensitivity and specificity of the method, was determined. Statistical analyses were performed using GraphPad Prism software, version 5 (GraphPad Software, Inc., La Jolla, CA, USA). Data were analysed by one-way analysis of variance followed by Tukey's post hoc test. $\mathrm{P}<0.05$ was considered to indicate a statistically significant difference.

\section{Results}

Comparison of panfungal PCR analysis and conventional culture. As shown in Fig. 1, the fungal ITS region was successfully amplified in blood specimens representing a single PCR product of $\sim 400$ base pairs. Sterile water and a fungus DNA sample were used as a negative and positive control, respectively (Fig. 1). In addition, GAPDH was included as an internal control, and no evident amplification inhibition was detected during the PCR reactions (Fig. 2). 
Table I. Panfungal PCR compared with conventional culture for the detection of IFDs.

\begin{tabular}{lccc}
\hline Analysis & Result & No. of patients & Percentage (\%) \\
\hline PCR & + & 33 & 71.7 \\
& - & 13 & 28.3 \\
\multirow{3}{*}{ Culture } & Total & 46 & 100.0 \\
& + & 9 & 19.6 \\
& - & 37 & 80.4 \\
& Total & 46 & 100.0 \\
\hline
\end{tabular}

PCR, polymerase chain reaction; IFD, invasive fungal disease.

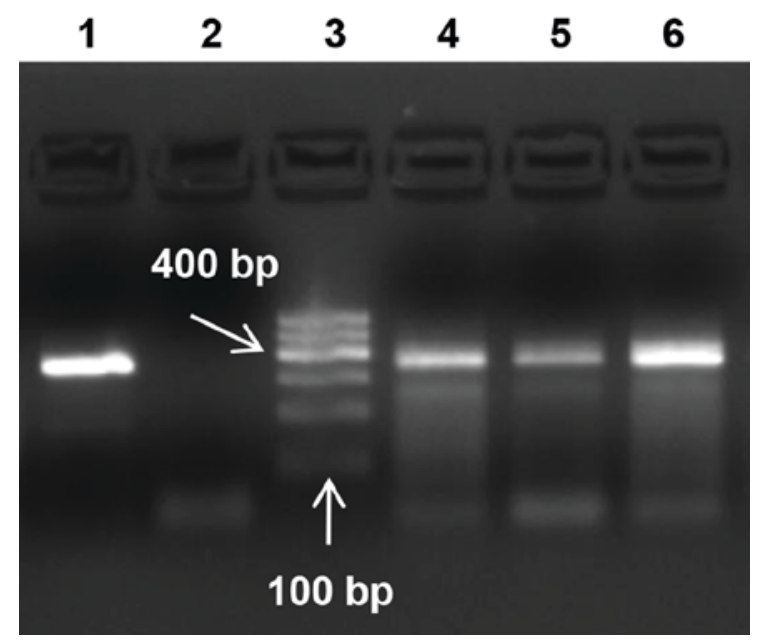

Figure 1. Agarose gel electrophoresis of internal transcribed spacer-polymerase chain reaction products demonstrating representative samples of positive species isolated from different samples of blood specimens. Lane 1 , positive control (Candida DNA); lane 2, negative control (sterile water); lane 3 , molecular size marker; lanes 4-6, positive species.

The study prospectively assessed the broad-range PCR analysis results as compared with the findings of conventional blood culture (Table I). Using conventional methods of blood culture, $19.6 \%$ (9/46) samples were found to be positive, with Candida albicans accounting for $>50 \%$ (5/9) of positive cases. In contrast to the blood culture results, a much higher percentage $(71.7 \% ; 33 / 46 ; \mathrm{P}<0.05)$ of enrolled patients tested positive in broad-range PCR analysis, which indicated an enhanced sensitivity of panfungal PCR compared with conventional culture in the diagnosis of IFDs in immunocompromised patients.

Fungal species identification by broad-range PCR and sequencing. The broad-range sequencing technique enabled the detection of 8 different fungal species in the 33 PCR-positive samples, including the 9 samples that were identified as positive by the culture method (Table II). Over half $(54.5 \%$; 18/33) of the samples from the PCR-positive group were identified as an unknown fungus. Consistent with the fungal species assignments by conventional culture, the most frequently observed species in the remaining samples included Candida albicans $(12.1 \% ; 4 / 33)$, Acremonium strictum $(12.1 \% ; 4 / 33)$ and
Table II. Type of fungus identified by broad-range sequencing in PCR-positive specimens.

\begin{tabular}{lcc} 
Species & No. of patients & Percentage $(\%)^{\mathrm{a}}$ \\
\hline Unknown fungus & 18 & 54.5 \\
Candida albicans & 4 & 12.1 \\
Acremonium strictum & 4 & 12.1 \\
Aspergillus fumigatus & 2 & 6.1 \\
Cladosporium oxysporum & 1 & 3.0 \\
Hypocrea & 1 & 3.0 \\
Trichosporon/Oidiodendron & 1 & 3.0 \\
Unknown bacteria & 1 & 3.0 \\
Undetermined source & 3 & 9.1
\end{tabular}

${ }^{\mathrm{a}}$ Out of 33 PCR positive specimens. PCR, polymerase chain reaction.

Aspergillus fumigatus $(6.1 \%$; 2/33). Furthermore, a variety of rare species, including Cladosporium oxysporum $(3.0 \%$; 1/33), Hypocrea $(3.0 \% ; 1 / 33)$ and Trichosporon/Oidiodendron $(3.0 \% ; 1 / 33)$, or other unknown bacteria $(3.0 \%$; 1/33) and undetermined sources $(9.1 \% ; 3 / 33)$ were observed in the remaining $21.1 \%$ of the total samples.

Characteristics of patients according to the EORTC/MSG criteria. According to the EORTC/MSG definitions, 30.4\% (14/46) of the included patients were classified as negative IFD cases, $21.7 \%(10 / 46)$ were classified as probable IFD cases and $47.8 \%(22 / 46)$ were classified as possible cases (Table III). Notably, if the patients were divided into PCR-negative and PCR-positive groups, the distribution patterns were significantly different in these two groups. The PCR-negative group consisted of $76.9 \%$ (21.7\% in total) negative IFD classifications, $23.1 \%$ (6.5\% in total) possible IFD classifications and $0 \%(0 \%$ in total) probable IFD classifications (Table III and Fig. 3). By contrast, there was a much lower level of negative IFD classifications (12.1\% within the group; $8.7 \%$ in total), a higher level of possible IFD classifications (21.2\% within the group; $12.1 \%$ in total) and significantly increased amount of probable IFD classifications (66.7\% within the group; $47.8 \%$ in total) in the PCR-positive group, as compared with the PCR-negative patients (Table III and Fig. 3). These findings highlighted the diagnostic value of panfungal PCR tests in distinguishing potential IFDs in immunocompromised patients.

Diagnostic performance of panfungal PCR in IFDs. The diagnostic performance of panfungal PCR according to the EORTC/MSG criteria of IFDs is presented in Table IV. In addition, ROC curve analysis was performed for panfungal PCR in IFD diagnosis (Fig. 4). Since no patients were classified as proven IFD cases according to the EORTC/MSG criteria in the present study, the diagnostic values of panfungal PCR was calculated under two different considerations, namely considering only probable or both probable and possible cases as positive IFD cases. Firstly, if only probable IFD was considered as positive IFD, panfungal PCR provided $100 \%$ sensitivity, $54.0 \%$ specificity, $67 \%$ PPV, 100\% NPV, 


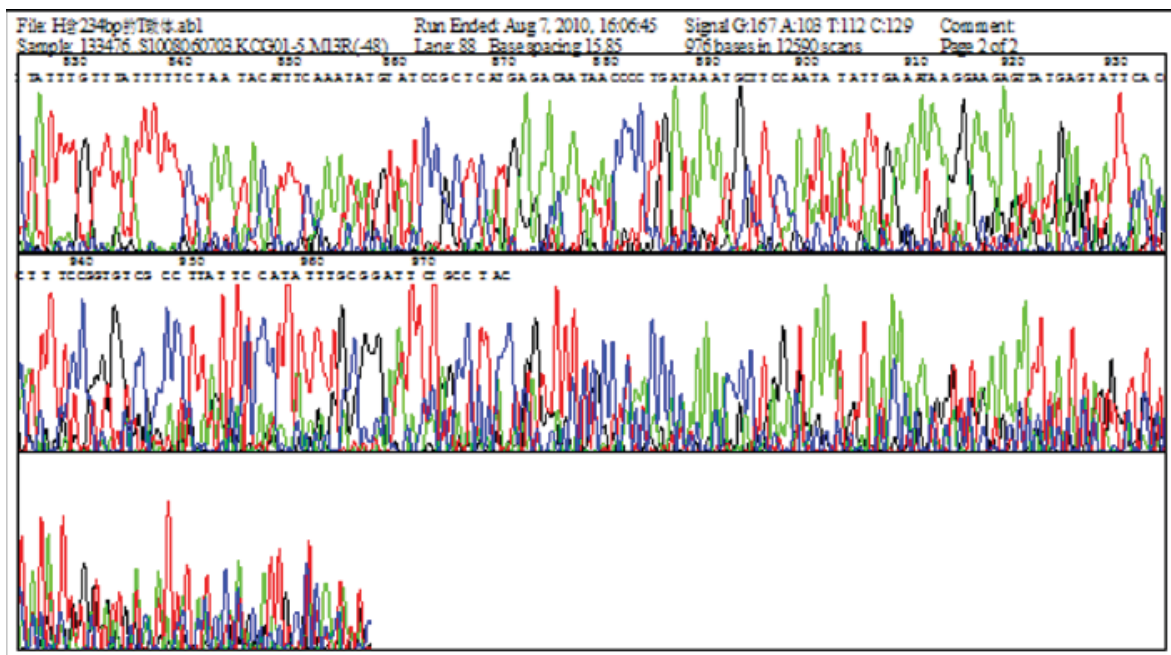

Figure 2. Sequencing analysis of GAPDH in the panfungal polymerase chain reaction. GAPDH was included as an internal control and no evident amplification inhibition was detected.

2.18 positive likelihood ratio (PLR) and 0 negative likelihood ratio (NLR). Secondly, when both probable and possible IFD were considered as positive IFD (Table V), the corresponding values were $93 \%, 71 \%, 91 \%, 77 \%, 3.25$ and 0.10 for sensitivity, specificity, PPV, NPV PLR and NLR. Furthermore, the areas under the ROC curves were 0.832 [95\% confidence interval (CI), 0.710-0.954; $\mathrm{P}<0.001]$ for the diagnosis of probable IFD (Fig. 4A) and 0.853 (95\% CI, 0.739-0.966; P<0.001) for the diagnosis of probable and possible IFD (Fig. 4B). In general, a larger OD value indicated a higher possibility of IFD diagnosis (Table V). The ROC curve analyses were used to determine the optional cut-off points (OD values), which were 271.5 (sensitivity, 82.8\%; specificity, 79.8\%; Fig. 4A) and 47.0 (sensitivity, $87.5 \%$; specificity, 71.4\%; Fig. 4B). Taken together, the presented evidence suggests that panfungal PCR assay may serve as a valuable adjunct to the EORTC/MSG criteria for IFD diagnosis.

\section{Discussion}

The aim of the present study was to assess the potential clinical benefit of molecular screening by PCR-based molecular diagnostic test in immunocompromised patients. A broad-range panfungal PCR assay was performed on blood specimens from immunocompromised patients, and the diagnostic performance of this technique for IFD was evaluated. In the current study, PCR test displayed an evidently higher sensitivity and similar diagnostic accuracy as compared with the conventional culture method. The PCR test demonstrated a sensitivity of $82.8 \%$ and a specificity of $79.8 \%$ for probable IFDs, or a sensitivity of $87.5 \%$ and a specificity of $71.4 \%$ for a combination of probable and possible IFDs, according to the EORTC/MSG criteria. By identifying the best cut-off points (OD values obtained from PCR analysis), the current study provided evidence that the PCR-based assay may serve as a quick and effective routine test for early diagnosis of IFDs in clinical laboratories.

Unlike other clinical samples, such as sputum, the blood samples from a healthy population are considered sterile (17). Thus, PCR-based analysis is more useful in detecting fungal
DNA from a blood sample due to its high sensitivity (18). However, blood specimens frequently contain a very small number of fungal cells; thus, the extraction methods used in the present study involved the lysis of a maximum number of fungal cells in order to yield a sufficiently large amount of DNA (19). Furthermore, modified purification techniques were also employed to remove contamination molecules that would interfere with the PCR reaction. The type of blood sample may also affect the sensitivity of the PCR assay (20). For instance, neutrophils from the whole blood samples are considered to contain greater amounts of Candida DNA, compared with serum assays (21). In order to achieve a maximum fungal DNA isolation, broad-range fungal PCR was used in the present study, and the DNA isolation and purification steps were optimized. In addition, the genomic DNA was extracted using the silica gel column method with lywallzyme solution and CTAB. The silica gel column method was designed to extract free fungal DNA, while the lywallzyme plus CTAB protocol was used to collect fungal DNA in the form of intact bodies. As a result, fungal DNA in the two formats was collected and amplified in the subsequent PCR assay.

According to the EORTC/MSG criteria, no proven IFD can be identified unless there is microbiological evidence from fluid samples (5). Besides, the blood culture method has various limitations, including low sensitivity, low specificity and lack of rapidity (4). In addition, numerous possible and probable IFD diagnoses are known to result from non-fungal causes $(22,23)$. In clinical practice, the severity of IFDs is not always correlated with the amount of fungal pathogen detected in the specimens (24). Considering the aforementioned reasons, developing a faster and more effective test is essential as an alternative method in the routine diagnosis of IFDs.

The current study calculated the diagnostic performance of panfungal PCR assay, including the sensitivity, specificity, PPV, NPV, PLR and NLR for probable and for probable plus possible patient populations. It was observed that panfungal PCR testing had an evidently higher sensitivity and similar accuracy in comparison with the conventional culture method. For instance, out of the 46 BMT recipient patients enrolled in 
Table III. Diagnosis of panfungal PCR in association with the EORTC/MSG criteria for invasive fungal disease.

EORTC/MSG diagnosis

\begin{tabular}{lcccc}
\cline { 2 - 3 } Result & Negative (\%) & Possible (\%) & Probable (\%) & Sum (\%) \\
\hline PCR positive & & & & \\
Count (\% of total samples) & $4(8.7)$ & $7(15.2)$ & $22(47.8)$ & $33(71.7)$ \\
$\%$ within the PCR positive group & 12.1 & 21.2 & 66.7 & 100 \\
PCR negative & & & & $13(28.3)$ \\
Count (\% of total samples) & $10(21.7)$ & $3(6.5)$ & $0(0.0)$ & 100 \\
$\%$ within the PCR negative group & 76.9 & 23.1 & 0.0 & $46(99)$ \\
Sum, count (\% of total) & $14(30.4)$ & $10(21.7)$ & $22(47.8)$ & \\
\hline
\end{tabular}

PCR, polymerase chain reaction; EORTC/MSG, European organization for research and treatment of cancer/mycoses study group.

Table IV. Diagnostic performance of panfungal polymerase chain reaction assay in immunocompromised patients in comparison with the EORTC/MSG criteria.

\begin{tabular}{lcc}
\hline Parameter & Probable $^{\mathrm{a}}$ & Probable and possible $^{\mathrm{a}}$ \\
\hline Sensitivity & 1.00 & 0.93 \\
Specificity & 0.54 & 0.71 \\
PPV & 0.67 & 0.91 \\
NPV & 1.00 & 0.77 \\
PLR & 2.18 & 3.25 \\
NLR & 0.00 & 0.10 \\
\hline
\end{tabular}

${ }^{\mathrm{a} A s}$ compared with the EORTC/MSG criteria. PCR, polymerase chain reaction; PPV, positive predictive value; NPV, negative predictive value; PLR, positive likelihood ratio; NLR, negative likelihood ratio; EORTC/MSG, European Organization for Research and Treatment of Cancer/Mycoses Study Group.

the present study, there were no cases of proven IFD (according to EORTC/MSG criteria), however, 22 cases (47.8\%) of probable and 10 cases $(21.7 \%)$ of possible infection were identified. These findings strongly indicate the current difficulty in obtaining an early diagnosis of IFDs in this patient population and the requirement for novel methods for early diagnosis. Following PCR analysis, the ratio of probable cases in the PCR-positive group and the negative cases in the PCR-negative group were greatly increased to 66.7 and $76.9 \%$, respectively. These results provide clear evidence that PCR assay has great diagnostic performance by sorting out people into two different groups, namely considering only probable or both probable and possible cases as positive IFD cases. PCR-positive patients appeared to have a higher potential of being confirmed with IFDs. Therefore, the use of panfungal PCR assay may also help to reduce the possibilities of misdiagnosis or missed diagnosis, and thus directly benefit other clinical decisions.

In addition to the blood culture method, the galactomannan (GM) antigen detection test is another diagnostic method extensively used for IFD diagnosis (25). The early diagnosis

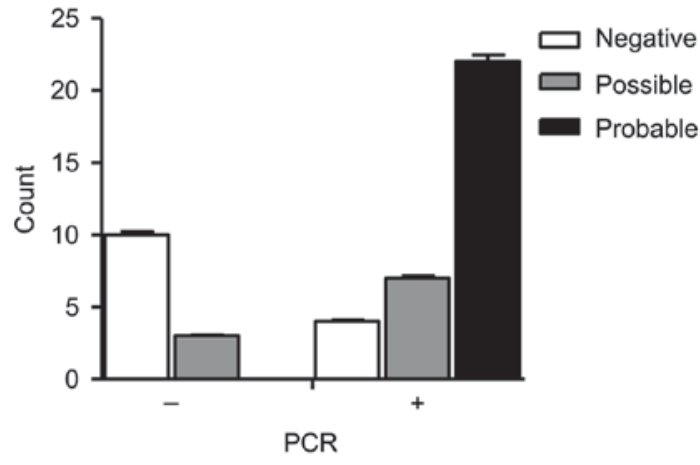

Figure 3. Distribution of negative, possible and probable invasive fungal disease, according to the European Organization for Research and Treatment of Cancer/Mycoses Study Group criteria, in the groups that presented negative or positive results in the panfungal PCR assay. PCR, polymerase chain reaction.
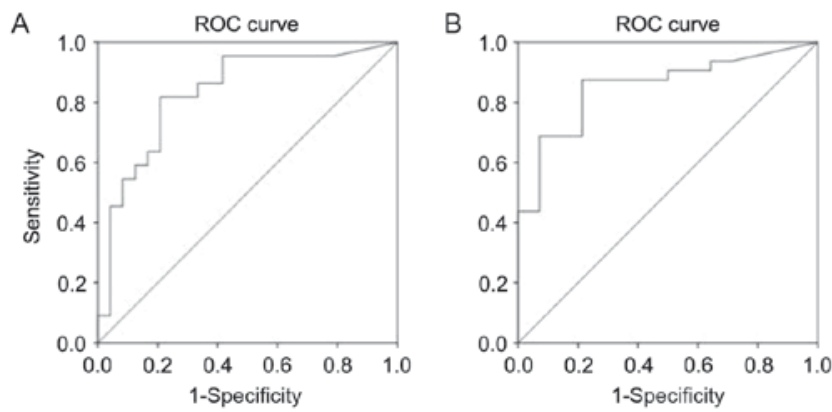

Figure 4. ROC curves for panfungal polymerase chain reaction assay results. The ROC curves were constructed as plots of sensitivity vs. 1-specificity when (A) probable IFDs were considered as positive and (B) both probable and possible IFDs were considered as positive. ROC, receiver operating characteristic; IFD, invasive fungal disease.

and treatment of IFD relies on the GM test, as well as a few other serological methods and radiological imaging. Although the GM antigen test has an acceptable sensitivity rate, it is also highly likely to produce false-positive results (26). In addition, this method does not distinguish between different fungal genera or species. For instance, an GM antigen test may be designed to 
Table V. Detailed PCR OD values from panfungal PCR analysis.

EORTC/MSG diagnosis (OD values)

\begin{tabular}{lrrc}
\cline { 2 - 4 } Result & Negative & Possible & Probable \\
\hline PCR positive & $254.52(\mathrm{n}=4)$ & $122.24(\mathrm{n}=7)$ & $2216.64(\mathrm{n}=22)$ \\
PCR negative & $13.1(\mathrm{n}=10)$ & $9.57(\mathrm{n}=3)$ & - \\
\hline
\end{tabular}

PCR, polymerase chain reaction; OD, optical density; EORTC/MSG, European Organization for Research and Treatment of Cancer/Mycoses Study Group.

specifically detect Aspergillus species, but also be cross-reactive to Penicillium chrysogenum and Paecilomyces antigens $(27,28)$. In contrast to the high specificity (71\%) of fungal PCR assay identified in the present study, the fact that the GM antigen detection test is not specific for IFDs is an important limitation of this method (29-31). Since little is known regarding the mechanisms of fungal pathogenesis, the clinical implication of the GM antigen detection test is more limited in comparison with other molecular methods, such as the PCR test and sequencing analysis. The latter methods, as demonstrated in the present study, were able to accurately identify the pathogens to the level of species. In addition, broad-range PCR assays can be designed for the detection of anti-fungal drug resistance, which is a growing concern (32).

The current study also identified two cut-off points (PCR-obtained OD values) based on ROC analysis. Although the accuracy remains to be confirmed by further studies, it is suggested that an OD value of $<47.0$ corresponds with negative IFD, an OD value between 47.0 and 271.5 corresponds with possible IFD, and an OD value of $>271.5$ corresponds with probable IFD. Therefore, it is proposed that, following confirmation from more independent experiments and clinical analyses, one set of PCR-determined OD values can be used as a guideline for assigning patients to certain risk groups in order to better determine their healthcare requirements.

In conclusion, the results of the present study suggested that the panfungal PCR method is a more sensitive and specific test compared with the conventional culture method. In order to satisfy the clinical requirement, it is suggested that the PCR results must be integrated in a clinical workup, including other validated markers, such as antigen detection, as well as direct examination and blood cultures. Thus, future studies should focus on the integration of these techniques into clinical decision-making.

\section{Acknowledgements}

This study was supported by grants from the Shanghai Health and Family Planning Commission (grant no. 20154Y0141) and the Shanghai Minhang District Science and Technology Commission (grant nos. 2016MHZ01 and 2017MHZ58).

\section{References}

1. de Pauw B: Contemplations on the challenge of invasive fungal disease. Clin Drug Investig 33 (Suppl 1): S57-S58, 2013.
2. Akan H, Antia VP, Kouba M, Sinkó J, Tănase AD, Vrhovac R and Herbrecht R: Preventing invasive fungal disease in patients with haematological malignancies and the recipients of haematopoietic stem cell transplantation: Practical aspects. J Antimicrob Chemother 68 (Suppl 3): iii5-iii16, 2013.

3. Drgona L, Colita A, Klimko N, Rahav G, Ozcan MA and Donnelly JP: Triggers for driving treatment of at-risk patients with invasive fungal disease. J Antimicrob Chemother 68 (Suppl 3): iii17-iii24, 2013.

4. Mikulska M, Furfaro E and Viscoli C: Non-cultural methods for the diagnosis of invasive fungal disease. Expert Rev Anti Infect Ther 13: 103-117, 2015.

5. Schelenz S, Barnes RA, Barton RC, Cleverley JR, Lucas SB, Kibbler CC and Denning DW; British Society for Medical Mycology: British society for medical mycology best practice recommendations for the diagnosis of serious fungal diseases. Lancet Infect Dis 15: 461-474, 2015.

6. Desoubeaux G, Bailly É and Chandenier J: Diagnosis of invasive pulmonary aspergillosis: Updates and recommendations. Med Mal Infect 44: 89-101, 2014.

7. Powers-Fletcher MV and Hanson KE: Nonculture diagnostics in fungal disease. Infect Dis Clin North Am 30: 37-49, 2016.

8. Cruciani M, Mengoli C, Loeffler J, Loeffler J, Donnelly P, Barnes R, Jones B, Klingspor L, Morton O and Maertens J: Polymerase chain reaction blood tests for the diagnosis of invasive aspergillosis in immunocompromised people. Cochrane Database Syst Rev: Cd009551, 2015.

9. Frías-de León MG, Acosta-Altamirano G, Duarte-Escalante E, Martínez-Hernández JE, Martínez-Rivera Mde L and Reyes-Montes Mdel R: Molecular markers: An important tool in the diagnosis, treatment and epidemiology of invasive aspergillosis. Cir Cir 82: 109-118, 2014 (In Spanish).

10. Halliday CL, Kidd SE, Sorrell TC and Chen SC: Molecular diagnostic methods for invasive fungal disease: The horizon draws nearer? Pathology 47: 257-269, 2015.

11. Hasseine L, Cassaing S, Robert-Gangneux F, Fillaux J, Marty P and Gangneux JP; PF-qPCR Study Group: High negative predictive value diagnostic strategies for the reevaluation of early antifungal treatment: A multicenter prospective trial in patients at risk for invasive fungal infections. J Infect 71: 258-265, 2015.

12. Cao G, Zhao Z, Peng Y, Ji Y, Sun J, Wei B and Liu L: The extraction of fungal genome DNA and the establishment of a broad-range fungal PCR. Jianyan Yixue 26: 773-778, 2011 (In Chinese).

13. Cao G, Zhao F, Liu Z, Hua L, Ji Y and Zhao Z: Clinical application of a fungal PCR assay to detect fungal DNA in sputum specimens. Guoji Jianyan Yixue Zazhi 33: 1635-1636, 2012 (In Chinese).

14. De Pauw B, Walsh TJ, Donnelly JP, Stevens DA, Edwards JE, Calandra T, Pappas PG, Maertens J, Lortholary O, Kauffman CA, et al: Revised definitions of invasive fungal disease from the European organization for research and treatment of cancer/invasive fungal infections cooperative group and the national institute of allergy and infectious diseases mycoses study group (EORTC/MSG) consensus group. Clin Infect Dis 46: 1813-1821, 2008.

15. White TJ, Bruns T, Lee S and Taylor JW: Amplification and direct sequencing of fungal ribosomal RNA genes for phylogenetics. PCR Protocols 18: 315-322, 1990.

16. Ao JH, Hao ZF, Zhu H, Wen L and Yang RY: Environmental investigations and molecular typing of Aspergillus in a Chinese hospital. Mycopathologia 177: 51-57, 2014. 
17. Jensen L, Jensen AV, Praygod G, Kidola J, Faurholt-Jepsen D, Changalucha J, Range N, Friis H, Helweg-Larsen J, Jensen JS and Andersen AB: Infrequent detection of Pneumocystis jirovecii by PCR in oral wash specimens from TB patients with or without HIV and healthy contacts in Tanzania. BMC Infect Dis 10: 140, 2010

18. Arishima T and Takezawa J: Use of PCR based diagnosis for common invasive fungal infections in the intensive care unit. Nihon Ishinkin Gakkai Zasshi 47: 283-288, 2006 (In Japanese).

19. Perfect JR: Fungal diagnosis: How do we do it and can we do better? Curr Med Res Opin 29 (Suppl 4): S3-S11, 2013.

20. Tattevin P, Revest M, Lefort A, Michelet $C$ and Lortholary O: Fungal endocarditis: Current challenges. Int J Antimicrob Agents 44: 290-294, 2014.

21. Gilchrist KB, Garcia MC, Sobonya R, Lipke PN and Klotz SA: New features of invasive candidiasis in humans: Amyloid formation by fungi and deposition of serum amyloid $\mathrm{P}$ component by the host. J Infect Dis 206: 1473-1478, 2012.

22. Zeng SY, Liu T, Meng WT and Chen YN: The significance of serum GM and BG antigens assay for invasive fungal infections in hematological malignancies patients. Zhonghua Xue Ye Xue Za Zhi 32: 43-46, 2011 (In Chinese).

23. Ram R, Gafter-Gvili A, Raanani P, Yeshurun M, Shpilberg O, Dreyer J, Peck A, Leibovici L and Paul M: Surveillance of infectious complications in hemato-oncological patients. Isr Med Assoc J 11: 133-137, 2009.

24. Ruhnke M: Antifungal stewardship in invasive Candida infections. Clin Microbiol Infect 20 (Suppl 6): S11-S18, 2014.
25. Kedzierska A, Kochan P, Pietrzyk A and Kedzierska J: Current status of fungal cell wall components in the immunodiagnostics of invasive fungal infections in humans: Galactomannan, mannan and (1->3)-beta-D-glucan antigens. Eur J Clin Microbiol Infect Dis 26: 755-766, 2007.

26. Fortún J, Martín-Dávila $\mathrm{P}$, Alvarez ME, Norman F, Sánchez-Sousa A, Gajate L, Bárcena R, Nuño SJ and Moreno S: False-positive results of Aspergillus galactomannan antigenemia in liver transplant recipients. Transplantation 87: 256-260, 2009.

27. White PL, Wingard JR, Bretagne S, Löffler J, Patterson TF, Slavin MA, Barnes RA, Pappas PG and Donnelly JP: Aspergillus Polymerase Chain reaction: Systematic review of evidence for clinical use in comparison with antigen testing. Clin Infect Dis 61: 1293-1303, 2015.

28. Sun WK, Zhang F, Xu XY, Shen YY and Shi Y: A systematic review of the accuracy of diagnostic test of serum galactomannan antigen detection for invasive aspergillosis. Zhonghua Jie $\mathrm{He} \mathrm{He}$ Hu Xi Za Zhi 33: 758-765, 2010. (In Chinese)

29. Springett GM: Novel pancreatic cancer vaccines could unleash the army within. Cancer Control 21: 242-246, 2014.

30. Jinushi M, Hodi FS and Dranoff G: Enhancing the clinical activity of granulocyte-macrophage colony-stimulating factor-secreting tumor cell vaccines. Immunol Rev 222: 287-298, 2008.

31. Dranoff G: GM-CSF-secreting melanoma vaccines. Oncogene 22: 3188-3192, 2003

32. Bhattacharya S: Early diagnosis of resistant pathogens: How can it improve antimicrobial treatment? Virulence 4: 172-184, 2013. 